\title{
EL «ROMANCE FAMILIAR» SALE DEL ARMARIO: LA FANTASÍA FEMENINA EN SÉVIGNÉ (JULIA BERKOWITZ)*
}

\author{
LEIRE ITUARTE PÉREZ \\ Universidad del País Vasco
}

Recibido/12/03/2012

Aceptado/24/05/2012

\section{Resumen}

Este ensayo aborda el estudio de Sévigné (Julia Berkowitz) (2004) de la cineasta catalana Marta Balletbó-Coll desde una perspectiva feminista que desgrana las particulares trasgresiones edípicas del filme respecto al «Melodrama materno» clásico. Su singularidad deriva de un «romance familiar» que se mueve en la dirección opuesta a la que sigue el Melodrama tradicional, en la medida en que la dramaturgia no trabaja para reprimir o canalizar la trasgresión femenina del tabú del incesto que implementa la función ideológica del Melodrama clásico, sino para sacarlo del armario y desvelar un triángulo edípico poco ortodoxo marcado por la inoperancia y la paulatina exclusión de la ley del padre.

Palabras Clave: Cine de mujeres, melodrama materno, romance familiar, teoría Queer, lógica edípica, trasgresión femenina.

\section{Abstract}

This essay tackles the study of Sévigné (Julia Berkowitz) (2004), by the Catalan filmaker Marta Balletbó-Coll, from a feminist perspective that deals with the specific

\footnotetext{
* Este ensayo es resultado de un proyecto de investigación sobre Melodrama y «Cine de mujeres» español contemporáneo que ha contado con la colaboración y financiación del Departamento de Educación, Universidades e Investigación del Gobierno Vasco.
} 
Oedipal transgressions of the film with regard to the classical «Maternal Melodrama». Its peculiarity derives from a «family romance» which moves in the opposite direction of the traditional Melodrama insofar as the drama does not work to repress or redirect the female transgression of the incest taboo carried by the ideological function of the classical Melodrama but to take it out of the closet and reveal an Oedipal triangle marked by the ineffectiveness and progressive exclusion of the Law of the father.

Keywords: Woman's film, «maternal melodrama, family romance, Queer theory, Oedipal logic, female transgression. 


\section{Sévigné (Julia Berkowitz), un romance singular}

Tras la buena acogida crítica de Costa Brava (Family Album) $(1995)^{1}$ y el batacazo de "Cariño, he enviado a los hombres a la luna...» (1998), la realizadora Marta Balletbó-Coll, a quien algunos comentaristas han definido como la Woody Allen catalana, se consolida como autora de un universo fílmico transgresor y muy personal gracias a Sévigné (Julia Berkowitz) (2004)², un Melodrama familiar que saca del armario el deseo lésbico reprimido de una mujer de edad madura sumida en el luto por el fallecimiento de su hija. Sévigné toma como trasfondo dramático el «romance edípico familiar» de una de sus protagonistas para deconstruir la melancolía femenina y llevar a escena la historia de amor entre dos mujeres: Julia Berkowitz (Anna Azcona), una ex actriz de teatro convertida en directora teatral de edad madura, atractiva, felizmente casada y bien acomodada que, sin embargo, vive apesadumbrada por el recuerdo de la muerte de su hija Tanit. Julia se encuentra dividida entre su presuntuoso marido Gerardo -un influyente crítico de teatro- y su amante Eduard Farelo, joven y atractivo ayudante de dirección teatral (Ignacio Basauri), cuando conoce a Marina -personaje con reminiscencias Woodyallenescas interpretado por la propia Balletbó-Coll- una dramaturga profesionalmente frustrada que trabaja en un estudio de televisión para poder sobrevivir pero que no ceja en su empeño por que alguien produzca su última obra teatral, adaptación de un drama materno-filial de época para dos personajes femeninos sobre la relación incestuosa entre la célebre aristócrata francesa Madame de Sévigné y su hija Madame de Grignan ${ }^{3}$. Tras las repetidas negativas del

1. Filme galardonado con el Premio especial de Calidad del Ministerio de Cultura, Premio Ciudad de Barcelona, Premio RNE Sant Jordi y el Premio del Público en los festivales Frameline (San Francisco), Outfest (Los Ángeles), Cineffable (París) y Un sguardo diverso (Milán).

2. Galardonada en 2006 con el Premio Nacional de Cine de Cataluña y con el Gran Premio del Jurado en el Festival Filmes de mujeres de Créteil.

3. La relación entre la marquesa de Sévigné y su hija Françoise-Marguerite, condesa de Grignan, se ha hecho célebre gracias al epistolario, hoy considerado una de las obras maestras literarias del XVII francés, en el que Madame de Sévigné plasmó el tortuoso amor por su hija. El genio de Sévigné fue reconocido además por el preciosismo y por la 
teatro público de Barcelona para programar su Sévigné, Marina consigue finalmente por mediación de Ignasi que Julia, tras no pocas reticencias, acceda a producir y dirigir la obra a condición de que el texto sea sometido a los cortes y cambios que ella estime necesarios. Julia se embarca en el proyecto de la Sévigné a pesar de la rotunda desaprobación de Gerardo, quien parece augurar el futuro romance al tratar de persuadir por todos los medios a su mujer para que no ponga en riesgo su brillante carrera como directora teatral haciéndose cargo de una obra que, según su criterio, será un flagrante fracaso de taquilla. En una de sus tentativas de persuasión para que Julia abandone el proyecto de la Sévigné le dirá: «No se puede dejar a dos mujeres solas en ese escenario inmenso del Teatro público, no se puede». El proceso de colaboración en torno a la reescritura y correcciones del texto durante su retiro en la casa de campo de Julia encenderá la mecha de la relación amorosa entre las dos protagonistas que terminará por confirmar las peores sospechas de Gerardo.

La intensa convivencia durante las diferentes fases de reescritura de la obra dará lugar a una relación cada vez más íntima que transcurre entre el intercambio de confidencias y el trabajo de corrección y edición del texto en el que Julia, que lleva la voz cantante, tomará las decisiones. Precisamente en el transcurso de dicha convivencia, Berkowitz, en una escena que mediante un primerísimo plano de la protagonista registra su profunda infelicidad, le confiesa a Marina:

Es esta sensación de no poder hacer nunca lo que te propones, de no hacer nunca lo que necesitas. En la vida todo va bien ¿no?, o parece que va bien. ¿Pero sabes que no recuerdo la última vez que soñé?, ¿Ni de qué sueño se trataba? Todo se resume en una única frase de Madame de Sévigné a su hija, una: 'Embarcada en la vida sin mi consentimiento'.

Pronto la voz en off de Marina revelará su fascinación por Julia: «Y ahora me encuentro diciendo sí a los cambios a los que hace una hora he dicho no y poniendo su embarcada como tema central de la obra. Me he enamorado de la Berkowitz $y$ de qué manera».

La confesión de Julia, en esta escena, se convierte en el primer indicio de su progresiva identificación con el personaje dramatúrgico de Madame de Sévigné. Identificación que irá incrementándose a medida que la protagonista

vívida recreación de la vida cortesana de sus escritos donde relataba con gracia y ligereza los cotilleos mundanos de la corte de Luis XIV. El propio Voltaire consideraba que era la primera persona en su siglo «en cuanto al estilo epistolar, y sobre todo para contar bagatelas con gracia». Sévigné escribió cerca de un millar de cartas a su hija (jamás pensó que serían publicadas tras su muerte) recogidas en un largo epistolario que se inaugura en febrero de 1671, después de la partida de la Marquesa de Grignan a Provenza, donde su esposo ocupaba el cargo de lugarteniente general del Rey. 
se sumerge en la lectura compulsiva de las cartas de amor que la aristócrata francesa escribió a su hija ausente. Su obsesión por conocer los detalles y pormenores de la relación incestuosa entre Madame de Sévigné y Madame de Grignan pronto se revela como una terapia de introspección personal que le permitirá ir indagando en los verdaderos sentimientos que le unieron a su difunta hija Tanit. En la reveladora escena en la que Julia recrimina a Marina su falta de valor para que el incesto entre madre e hija se haga explícito en el texto terminará confesándole: «Me da igual lo que sintió Madame de Sévigné por su hija, lo que me interesa saber es qué sentí yo por la mía». Más tarde, cuando en el transcurso de una de las escenas íntimas que transcurren en el interior de la casa de campo Marina le pregunta: « $i$ Te enamoraste de tu hija?» Julia le revela: «Yo no soy consciente de haberla querido así pero no me la puedo sacar de la cabeza. Marina, no puedo dormir, no tengo vida...», antes de explotar en un ataque histérico de ansiedad, en el que desesperada le confiesa:»...no puedo llorar, desde la muerte de Tanit...no puedo llorar...».

Este sumario recuento de la trama edípica de Sévigné (Júlia Berkowitz) en 2004, es suficiente para ilustrar que el filme constituye un caso singular en su adscripción al género melodramático. Particularidad que deriva ya no sólo de la progresiva visibilidad que la trasgresión femenina del tabú del incesto ocupa en la trayectoria dramática del relato fílmico sino de la propia incapacidad de la narrativa para la debida canalización y dosificación del «excedente» del deseo femenino/materno que implementa la función ideológica del Melodrama clásico. Es más, la progresión narrativa de este singular «romance familiar» se mueve, precisamente, en la dirección opuesta a la que sigue el Melodrama tradicional en la medida en que la dramaturgia no trabaja para reprimir o canalizar la amenaza del incesto materno-filial -el dispositivo central de una trasgresión femenina que aquí comparece junto a la necrofilia y la homosexualidad- sino para sacarlo del armario y desvelar un triángulo edípico poco ortodoxo marcado por la inoperancia y la paulatina exclusión de la ley del padre.

\section{Transgresiones edípicas en el «melodrama materno»}

Para valorar el alcance, desde una perspectiva feminista, de la naturaleza trasgresora de este filme, respecto a los parámetros del Melodrama tradicional, es preciso retroceder un poco en la historiografía de un género que, incluso en su modalidad más clásica, aparece marcado por la ambivalencia ideológica. En la célebre disección del «Cine de mujeres» hollywoodiense de su The Desire to desire (The Woman's Film of the 1940s), Mary Ann Doane señalaba a propósito de la configuración dramatúrgica del pathos como emoción central 
del melodrama clásico que la mujer casi siempre cumple una función dramática que la convierte en depositaria de todas las desgracias que acarrea el drama. Atrapada en un escenario familiar claustrofóbico y opresivo la protagonista, vulnerable, impotente e indefensa, suele enfrentarse al peligro y la amenaza de un drama cuya magnitud le sobrepasa. Desde esta perspectiva, la autora añade con acierto que el hecho de que en el «Cine de mujeres» ${ }^{4}$, el efecto patético casi siempre recaiga sobre la victimización de las mujeres hace del melodrama clásico un género cuando menos emblemático y representativo de los conflictos que derivan de la difícil posición cultural de las mujeres en el contexto patriarcal. Conviene no olvidar, sin embargo, como puntualiza Pam Cook parafraseando a Laura Mulvey que, tratándose de un género mayormente orientado a una audiencia femenina, "...para seducir a la espectadora femenina, el melodrama debe cuando menos proponer la posibilidad del deseo femenino... $»^{5}$. En este sentido, añade Cook, en la medida en que el «Cine de mujeres» demanda una protagonista femenina central que es el sujeto activo del deseo en lugar de objeto del deseo masculino, como suele ser habitual en el cine clásico, se convierte en un género virtualmente transgresor, máxime cuando la posibilidad del deseo femenino suele presentar una dificultad adicional para la narrativa clásica que difícilmente puede contenerse

4. Mary Ann Doane apuntala su conceptualización del «Cine de mujer» como una modalidad del cine de Hollywood que abarca un arco cronológico que comienza en el cine mudo y termina en los años 60, con su máxima concentración de filmes durante la década de los 30 y 40. Véase al respecto: Doane, Mary Ann. The Desire to Desire (The Woman's Film of the 1940s). Indiana University Press, Bloomington\&Indianapolis, 1987.pp.73

Aunque éste no sea lugar para abordar los pormenores historiográficos y dificultades conceptuales que han acompañado a la catalogación del «Cine de mujeres» como género por derecho propio ni para el debate en torno a las fugas e intersecciones entre el Melodrama y el «Cine de mujeres», conviene recordar, que, como señalan Mercer y Shingler $(2004,35)$, la equiparación entre estos dos términos se hizo efectiva en los años 80 una vez que el melodrama fue repensado como un género mayormente dirigido a una audiencia femenina. Para un sumario recuento historiográfico de la categorización genérica del Cine de mujeres véase: MERCER, John \& SHINGLER, Martin. Melodrama, (Genre, Style, Sensibility. Short Cuts, Wallflower, London/New York, 2004, pp. 33-37.

Si bien es cierto que hace mucho ya que el Cine de mujeres, reconocido como un género por derecho propio asimilable al Melodrama, prescindió de las comillas, hemos decidido mantenerlas por una cuestión de coherencia terminológica que quiere hacer justicia a la naturaleza fronteriza de una etiqueta que en el caso que nos ocupa $-\mathrm{y}$ tras la cada vez más numerosa incorporación de la mujer al ámbito de la producción cinematográfica- no sólo alude a un género clásico mayormente destinado a una audiencia femenina sino a un cine realizado por mujeres.

5. La traducción es de quien escribe: «...in order to appeal to a female spectator, melodrama must first posit the possibility of female desire...», en COOK, Pam. "Melodrama and the Women's Picture», en ASPINALl, Sue \& MURPHY. Robert (eds). Gainsborough Melodrama, BFI Dossier, No 18, British Film Institute, 1983, p.17. 
o canalizarse si no es mediante la problematización del punto de vista de la protagonista, léase, mediante la tradicional puesta en escena de algunos de los escenarios paradigmáticos de la feminidad como la melancolía, la paranoia, el masoquismo o la histeria, muy habituales en este tipo de filmes.

La argumentación de Cook sintoniza, desde una perspectiva feminista, con la reivindicación que, años antes, teóricos como Thomas Elsaesser ${ }^{6}$ o Geoffrey Nowell-Smith ya habían hecho del melodrama por su potencial ideológicamente subversivo ${ }^{7}$. Según este último:

La importancia del melodrama (...) reside precisamente en su fallo ideológico. Puesto que no puede acomodar sus problemas (...) sino que los exhibe abiertamente en su desvergonzada naturaleza contradictoria, abre un espacio que la mayoría de las formas de Hollywood han clausurado estudiosamente ${ }^{8}$.

Precisamente en esa misma dirección Mary Ann Doane presentaría, años más tarde, una definición más ajustada para el «Cine de mujeres» en base a una categorización del género que, más allá de la importancia de su orientación a una audiencia femenina, ponía el énfasis en la puntualización de una serie

6. Cfr. ElSAesser, Thomas, «Tales of Sound and Fury (Observations on the Family Melodrama)». Monogram, $\mathrm{n}^{\circ}$ 4, 1972, pp.2-15.

7. Como es sabido el debate feminista de los años 70/80 en torno al Melodrama también ponía en primer término la cuestión ideológica, esto es, el modo en el que finalmente el «Cine de mujeres» gestionaba la trasgresión femenina. Sin embargo, el posicionamiento de la Teoría fílmica feminista a este respecto no dejaba de estar marcado por la ambivalencia y el escepticismo derivados de una paradoja que apuntaba al hecho de que si bien las consecuencias de dicha trasgresión femenina siempre se volvían narrativamente contra la mujer -mediante estrategias clásicas como el castigo, el sufrimiento, la impotencia frente a los avatares del destino y las reiteradas represalias sociales que recaen sobre ella- la narrativa llevaba implícita una contradicción interna que generaba un problema irresoluble, un exceso imposible de contener que obstaculizaría el cierre narrativo convencional. Así en el célebre ensayo de Laura Mulvey «Notes on Sirk and Melodrama», la prometedora idea inicial que abogaba por la contradicción ideológica como componente utópico inherente al melodrama -cuya función primaria consistiría, precisamente, en dar salida a las inconsistencias ideológicas que vendrían a demostrar que ninguna ideología puede aspirar a la totalidad-desemboca en un balance final teñido de pesimismo: «Si el melodrama ofrece una evasión de fantasía para las mujeres identificadas en la audiencia, la ilusión aparece tan fuertemente marcada por trampas reconocibles, reales y familiares, que la evasión está más cerca de un sueño diurno que de una historia razonable». En MulveY, Laura. «Notes on Sirk and Melodrama» (1977/78), en GLEDHILL, Christine (ed). Home is Where the Heart is (Studies in Melodrama and the Woman's Film. BFI, London, 1987, pp. 75-79.

8. La traducción es de quien escribe: «The importance of melodrama (...) lies precisely in its ideological failure. Because it cannot accommodate its problems (...) but lays them open in their shameless contradictoriness, it opens a space which most Hollywood forms have studiously closed off», en NowELL-SMITH, Geoffrey. «Minelli and Melodrama». Screen, 18, 2, 1977, p. 114 .

Feminismo/s 19, junio 2012, pp. 13-27 
de constantes que, a pesar de la diversidad de los filmes englobados bajo la célebre etiqueta, venían a trasgredir los códigos patriarcales del cine clásico. Entre éstas destacaban el protagonismo femenino dominante, ya señalado por Cook, el acceso de la protagonista a ciertos privilegios tradicionalmente masculinos como la enunciación del discurso fílmico y el punto de vista, léase, el ejercicio escópico de la mirada femenina en la diégesis, la centralidad y problematización de la subjetividad femenina así como el tratamiento dramatúrgico de problemas y ansiedades propias del subconsciente colectivo de las mujeres y cómo no -de nuevo en consonancia con Cook- la puesta en escena del deseo femenino. Así las cosas, no es de extrañar que el «Cine de mujeres» se haya convertido para la Teoría feminista del cine en uno de los géneros más interesantes de cara a la virtualidad utópica de una transgresión femenina en el cine clásico. Desde una perspectiva feminista, la ambivalencia ideológica y su orientación a una audiencia mayoritariamente femenina quedan resaltadas además como características centrales de un género que constituye un laboratorio muy fértil para el estudio de los procesos de inscripción de la subjetividad y el deseo femeninos que configuran los modos de ver de las mujeres en el cine.

Es significativo que en el estudio de Doane, el «Melodrama materno» se configure, precisamente, como el subgénero más representativo del «Cine de mujeres $»^{9}$ por ser aquél que mejor vehicula el pathos melodramático y por tratarse de aquella modalidad que al reproducir los escenarios de separación y pérdida que escenifican el sufrimiento y sacrificio materno, constituye también, la variante más representativa del recurso lacrimógeno del Melodrama. El pathos que recae sobre la mujer en esta subcategoría del «Melodrama familiar» suele ir ligado a la narrativa de la dramática pérdida y/o separación de un hijo o hija con la consiguiente configuración de un escenario subliminal que asocia a la condición femenina la melancolía, la pérdida, el hueco o la carencia - a la postre, la castración-. Dicha pérdida suele ser correlativa, además, a la progresiva clausura de la posibilidad del deseo y la sexualidad femenina -al menos en los límites que impone la ley patriarcal- puesto que como refiere Doane en el «Melodrama materno» clásico «...el pathos es generado precisamente por una situación en la que el amor materno se convierte en signo de la imposibilidad del deseo femenino ${ }^{10}$. En el tratamiento diegético de la figura materna en este tipo de filmes suele ser habitual, por tanto, que la representación de la mujer vaya unida subliminalmente a su incompatibilidad

9. Para una revisión feminista de los pormenores del «Melodrama materno» en el marco del «Cine de mujeres» véase: Doane, Mary Ann. Op. Cit., pp. 70-95.

10. Ibid., p.94. 
con una sexualidad femenina «normativa». El deseo femenino, en todo caso, cuando no es totalmente suprimido de la narración, suele comparecer bajo el fantasma del tabú del incesto y la consiguiente amenaza de la estigmatización y penalización de la figura materna Como señala Doane

... a este presunto 'fallo' o 'defecto' en la sexualidad femenina -el deseo maldireccionado de la mujer, su insistencia en esforzarse por el objeto 'equivocado'- le es dada una representación extensiva en los 'melodramas maternos'. El deseo materno se revela frecuentemente como activamente resistente al desarrollo de la historia de amor ${ }^{11}$

En tanto que variante posmoderna del «Melodrama materno» tradicional, Sévigné trasgrede esta tendencia a clausurar la posibilidad de la sexualidad femenina, en la medida en que el deseo edípico materno-filial entre Julia y su difunta hija sale del armario, precisamente, gracias a la intervención del deseo homoerótico femenino entre las dos protagonistas, esto es, gracias a una aventura amorosa que aquí cumple una función ritual que saca a la luz un «romance familiar» largamente reprimido. Y lo que es más, este singular reducto femenino, un triángulo edípico configurado por tres mujeres donde, como decíamos, la figura paterna va quedando progresivamente ausente, constituye una nueva posibilidad para el deseo femenino y una alternativa al escenario edípico del «romance familiar» en el Melodrama tradicional. Escenario que, como es sabido, garantiza cuestiones esenciales en torno a la identidad, la subjetividad e individuación del sujeto y los límites entre el yo y el otro que instaura la ley patriarcal. Por ello, como recuerda Nowell-Smith en su revisión de Melodrama Hollywoodiense,

El melodrama representa, muchas veces con una extraña literalidad, el 'romance familiar' descrito por Freud -esto es, el escenario imaginario que escenifican los niños en relación a su paternidad, la pregunta y la respuesta a la pregunta: ¿De quién soy hijo?- (...) Además de por los problemas de los adultos, particularmente las mujeres, en relación a su sexualidad, el melodrama de Hollywood también se preocupa fundamentalmente por los problemas infantiles de crecer en una identidad sexual dentro de la familia, bajo el eje de una ley simbólica que el padre encarna ${ }^{12}$.

11. Ibíd., p. 92.

12. La traducción es mía: «Melodrama enacts, often with uncanny literalness, the 'family romance' described by Freud -that is to say the imaginary scenario played out by children in relation to their paternity, the asking and answering of the question: whose child am I (...) In addition to the problems of adults, particularly women, in relation to their sexuality, the Hollywood melodrama is also fundamentally concerned with the child's problems of growing into a sexual identity within the family, under the aegis of a symbolic law which the Father incarnates", en NOWELL-SMITH, Geoffrey. Op. Cit., p. 116.

Feminismo/s 19, junio 2012, pp. 13-27 
No deja de ser significativo que según la teoría freudiana, recuerda NowellSmith, la fantasía del «romance familiar» en los casos de neurosis funcione como mecanismo para la gestión de las ansiedades que se generan durante las últimas fases del complejo de Edipo. La fiel reproducción de dicha fantasía en el «Melodrama familiar» hace del incesto un motivo recurrente -más o menos subliminal- que tiende a comparecer como amenaza contra la estabilidad familiar y la ley del padre, precisamente en la medida en que la función primaria del «romance familiar» freudiano no es otra que la regulación del tabú del incesto. El «excesivo» deseo femenino del «Melodrama materno», en tanto que uno de los leitmotivs más recurrentes de la trasgresión femenina que vehicula este género, siempre lleva implícita la amenaza de una disfunción en la implementación de la ley patriarcal de la diferencia ${ }^{13}$. No es raro, por tanto, que en esta modalidad del «Melodrama familiar» el deseo incestuoso, en tanto que una modalidad del deseo femenino que debe permanecer reprimido o cuando menos debidamente dosificado y canalizado, aparezca subliminalmente codificado como un deseo excesivo desviado de la norma. Huelga decir que la función ideológica del Melodrama tradicional consiste, precisamente, en regular y canalizar las inestabilidades y amenazas de ese excedente del deseo femenino con el objeto de apuntalar la figura materna de acuerdo con los parámetros del escenario edípico que prescribe el patriarcado.

\section{Oedipus interruptus}

En Sévigné esa configuración de un nuevo paisaje edípico femenino que progresivamente clausura la intervención de la ley del padre no prescinde, sin embargo, de la tradicional representación histérica del cuerpo femenino - en tanto que superficie atravesada por «el retorno de lo reprimido»- que nos remite a la problemática representación de una sexualidad femenina trasgresora pero profundamente marcada por la represión de la orientación homosexual del deseo de la protagonista. La incapacidad de Julia para llorar el dolor de su pérdida -tal y como ésta le revela a Marina- se presenta aquí como síntoma histérico de una frigidez heterosexual femenina ${ }^{14}$ resultante de la larga

13. La renuncia materna, después de todo, es el prerrequisito ineludible para la individuación del sujeto. Como recuerda Julia Kristeva: «...la pérdida de la madre es una necesidad biológica y psíquica, el primer jalón de la emancipación. El matricidio es nuestra necesidad vital, condición sine qua non de nuestra individuación con tal de que suceda de manera óptima y pueda ser erotizado... EN KRISTEVA, Julia. Sol negro. Depresión y melancolía. Monte Avila Editores Latinoamericana, Venezuela, 1997, p. 30.

14. Recordemos con Thomas Elsaesser que «...el pathos (...) es frecuentemente utilizado en los melodramas para explorar la represión psicológica y sexual de los personajes (...) 
represión de un deseo incestuoso y homosexual. Represión que se revela, a lo largo del filme, como la fuente primigenia del estupor melancólico de la protagonista que apunta, más allá del duelo por la muerte de su hija Tanit, a la doble pérdida y represión de una modalidad del deseo femenino -ya no sólo incestuoso, sino homosexual- doblemente proscrita por el patriarcado.

En este sentido se puede afirmar que Sévigné escenifica, de un modo paradigmático, la equiparación entre la sexualidad femenina y la melancolía que la Teoría Queer atribuye al engranaje edípico, en la evolución de la sexualidad infantil femenina descrita por Freud. En su crítica al heterosexismo freudiano de su celebérrima Gender Trouble ${ }^{15}$ Judith Butler desgrana los pormenores de dicho engranaje para concluir que la melancolía se presenta como una dolencia poco menos que consustancial a la consolidación de un escenario sexual femenino ${ }^{16}$ que en la teoría freudiana se apuntala sobre la premisa de una represión doble: la del objeto y la modalidad homosexual del deseo femenino. Butler recuerda que, en dicha teoría, la orientación incestuosa heterosexual que marca el complejo de Edipo positivo en la niña, tiene como prerrequisito el tabú de la homosexualidad contra la extremada cercanía materno-filial que caracteriza al complejo negativo de Edipo femenino, antes de que con el advenimiento del complejo de castración -y la supuesta envidia fálica- el deseo homosexual de la pequeña sea convenientemente transferido a la figura paterna. La secuencia edípica femenina revela así, como subraya esta autora, una trayectoria sustancialmente más ardua que la del niño, dado que el desarrollo normativo de la feminidad presenta como premisa la «incorporación»

la impotencia masculina y la frigidez femenina». La traducción es mía: «...pathos (...) is frequently used in melodramas to explore psychological and sexual repression (...) male impotence and female frigidity», en ELSAESSER, Thomas. Op. Cit., p. 67.

Es relevante mencionar aquí el hecho de que, como demuestra una de las primeras escenas de convivencia doméstica entre Julia y Gerardo, estos no comparten dormitorio.

15. Para una revisión más pormenorizada de la asociación entre feminidad y melancolía en el marco de la crítica butleriana al heterosexismo freudiano puede consultarse su Gender Trouble (Feminism and the Subversión of Identity. Routledge, Londres/Nueva York, 1999 , pp.73 y ss.

16. En esa misma línea recuerda Kaja Silverman que el diagnóstico de la melancolía que Freud desarrolla en su célebre ensayo «La aflicción y la melancolía» ofrece «...el más escalofriante recuento de una condición que puede ser patológica para el sujeto masculino pero representa la norma del sujeto femenino -la condición de un narcisismo negativo que frustra sus relaciones tanto con ella misma como con su cultura». La traducción es mía: «... the most chilling account of a condition which may be pathological for the male subject, but represents the norm to the female subject -the condition of negative narcissism which blights her relations both with herself and her culture», en SILVERMAN, Kaja. The Acoustic Mirror (The Female Voice in Psychoanalysis and Cinema). Indiana University Press, Bloomington/Indianapolis, 1988, p.147. 
de una pérdida múltiple difícil de restituir que se configura como antesala de la melancolía femenina: junto a la pérdida del objeto materno primario y la renuncia de la modalidad homosexual del deseo femenino ${ }^{17}$ la niña habrá de superar, además, el traumático reconocimiento de la carencia de un órgano ajeno a su anatomía -el excedente fálico de su hermanito o de su padre- que en la secuencia edípica cumpliría la función de desencadenante de las sucesivas pérdidas que le son necesarias para su entrada en el buen puerto de la feminidad. Como recuerda Luce Irigaray,

A diferencia del niño (...) la pequeña toma a su madre como primer objeto amoroso y también como punto de referencia privilegiado de identificación para su 'ego' y su sexo. De hecho, si siguiéramos todas las implicaciones del discurso de Freud, después de que la pequeña descubre su propia castración y la de su madre -su 'objeto', la representación narcisista de todos sus instintos- no le quedaría otro recurso que la melancolía ${ }^{18}$

El corolario melancólico de este cúmulo de carencias, pérdidas irreparables y castraciones inconfesables de las que da cuenta la trama freudiana sobre la evolución de la sexualidad infantil femenina sería, como afirma Butler, que «la melancolía es así una norma psicoanalítica para las mujeres; una norma que descansa en su ostensible deseo de tener un pene, un deseo que, convenientemente, no puede ser reconocido como tal ${ }^{19}$. En la misma línea Julia Kristeva sentencia que nunca es redundante insistir en que,

Si ya el descubrimiento de su vagina invisible exige a la mujer un inmenso esfuerzo sensorial (...) el pasaje al orden simbólico al mismo tiempo que el pasaje a un objeto sexual de sexo distinto al del objeto materno primordial, representa una elaboración gigantesca para la cual una mujer necesita un potencial psíquico superior al exigido al sexo masculino (...) su costo es esa propensión a celebrar ininterrumpidamente el duelo problemático del objeto perdido... 20

En este sentido podemos afirmar que en Sévigné la narrativización del engranaje melancólico y las dificultades a las que el escenario edípico tradicional

17. Es precisamente en la intersección entre el complejo negativo de Edipo femenino y el complejo positivo donde autoras como Kaja Silverman o Luce Irigaray localizan la conexión entre feminidad y melancolía. Ibíd., p. 155.

18. La traducción es mía: «Unlike the boy (...) the little girl takes her mother as her first object of love and also as her privileged indentifcatory reference point for her 'ego' as well as for her sex. In point of fact, if all the implications of Freud's discourse were followed through, after the little girl discovers her own castration and that of her mother - her 'object', the narcissistic representative of all her instincts -she would have no recourse other than melancholia». Ibíd., p.155.

19. BUtLer, Judith. Op. Cit., p. 88.

20. Kristeva, Julia. Op. Cit., p. 32. 
somete a esa «otra» modalidad del deseo femenino trasgrede las convenciones del «Melodrama familiar» tradicional. Como decíamos, el filme desarrolla una trayectoria narrativa que sigue un recorrido inverso a la lógica discursiva del «Melodrama materno» clásico desde el momento en que la progresión narrativa se moviliza como interrupción e inversión de la trayectoria edípica dramatúrgica convencional ${ }^{21}$ para sacar del armario precisamente aquello que la función ideológica del «melodrama materno» tradicional trata de reprimir o canalizar, léase la abierta problematización y ambivalencia de la sexualidad femenina, la ritualización del deseo materno y la con-fusión de identidades. En esta trasgresión de la lógica edípica de la narrativa clásica encontramos un retroceso simbólico al escenario pre-edípico femenino donde el espectro de la relación madre-hija que pone en primer término otra modalidad del deseo femenino se enmarca en un escenario idílico de confusión visiblemente alejado de la imaginería «siniestra» de lo abyecto-femenino que suele comparecer en el discurso patriarcal de la feminidad ${ }^{22}$. Aquí encontramos también una eficaz estrategia que pone en práctica la narrativización del Oedipus Interruptus propuesto por Teresa de Lauretis donde la duplicidad del deseo femenino se mueve «con y contra la narración, para representar no sólo el poder del deseo femenino, sino su duplicidad y ambivalencia (...) -puesto que, según esta autora-, 'el cine de mujeres' debe encarnar y sacar a la luz la contradicción del deseo femenino.» ${ }^{23}$

21. No olvidemos que como recuerda Teresa de Lauretis: «Toda narración, en su avance hacia la resolución y en su retroceso al momento inicial, al paraíso perdido, está impregnado de lo que se ha denominado la lógica edípica -la necesidad intima de 'drama'- su sentido de un final 'inseparable del recuerdo de la pérdida y la reconquista del tiempo» en DE Lauretis, Teresa. Alicia ya no (Feminismo, Semiótica, Cine). Cátedra, Madrid, 1992, p. 199. Lo relevante para la teoría feminista es, claro está, el hecho de que toda narración hegemónica obedece a la lógica edípica del deseo de un sujeto arquetípico masculino que relega a mujer a los márgenes del cuadro narrativo.

22. El mérito y novedad de este filme radicaría, precisamente, en un tratamiento bien distinto de la «siniestra» codificación de lo abyecto-femenino materno. En Sévigné, el tratamiento del exceso materno y la con-fusión de identidades entre sujeto-objeto, entre madre e hija es de una índole bien distinta. El propio Gerardo -que aquí encarnaría la figura maquiavélica patriarcal- sugerirá a Marina que rehaga el texto de la Sévigné haciendo de esta con-fusión de identidades femeninas el objeto central del drama: «... lo del jansenismo no cuela ni con vaselina (...) La Sévigné huérfana desde niña, lo único que quiere es una madre y la Grignan, por otra parte, se pasa la vida pariendo. Sólo cuando las dos mujeres entienden que de lo que se trata es de intercambiar sus roles pueden llegar a entenderse». De igual manera Marina le propondrá a Julia erradicar el tema del jansenismo para centrarse en el intercambio de roles entre Madame de Sévigné y Madame de Grignan: "Madre e hija se amaron en Grignan, Provenza, cuando la hija se dio cuenta de que su papel era el de ser la madre de su propia madre».

23. De Lauretis, Teresa. Op. Cit., p.246.

Feminismo/s 19, junio 2012, pp. 13-27 
Ya hemos referido que la represión sexual de Julia, al igual que su inconsolable repliegue melancólico en el luto por la muerte de Tanit, tan sólo podrá resolverse una vez que ésta acceda a reconocer, como Madame de Sévigné, cuál ha sido la naturaleza de sus sentimientos por su hija y cuál es la verdadera orientación de su deseo. El curso dramatúrgico del pathos femenino trabaja, precisamente, para fomentar la anagnórisis de la protagonista mediante una paulatina regresión/trasgresión de la lógica edípica del relato que simbólicamente devolverá a Julia a un estadio pre-edípico de la idílica relación entre madre e hija. Durante las últimas fases de edición del texto, Berkowitz, cada vez más sumergida en la vertiente más carnal y sexual del epistolario entre Sévigné y su hija, convencerá a Marina para viajar hasta Grignan, una de las localizaciones francesas en las que madre e hija se amaron. Allí, en la habitación del hotel donde se alojan, Julia Berkowitz, tras haber accedido a interpretar al personaje de Sévigné -el colofón final de la identificación entre los dos personajes femeninos ${ }^{24}$-, le pedirá a Marina que reproduzca con ella la escena en la que Madame de Sévigné llora desesperadamente. «La quiero hacer ahora, quiero saber si puedo llorar. ¿Quieres hacerla conmigo? Haz de hija» le dice a Marina. En una escena tan cargada de sensualidad como de dolor y reconocimiento de su mutuo deseo, ambas mujeres reproducen, entre titubeos, la escena en la que Madame de Sévigné llora desconsoladamente por el amor y la «pérdida» de su hija. La escena que revivirá simbólicamente el amor incestuoso y reprimido entre madre e hija encarna al tiempo el reconocimiento y la consumación feliz del deseo femenino entre las dos mujeres, entrelazadas entre besos, caricias y abrazos y la explosión del llanto histérico y desesperado de Julia Berkowitz, por fin libre.

24. La progresiva confusión de identidades entre la protagonista de la diégesis -Julia Berkowitz- y la de la ficción dramatúrgica -Madame de Sévigné- cristalizará en esta escena donde, mediante la teatralización de otra escena de ficción, ambos personajes, finalmente, se funden. La escena constituye el momento culminante del juego constante de identificación entre los dos escenarios que configuran el doble marco de referencia de la ficción dramática -el de la diégesis fílmica y el de la ficción dramatúrgica de la Sévigné-finalmente fusionados. Un juego metadiscursivo que es ilustrativo de un proceso de somatización textual, esto es, de la utilización del cuerpo textual diegético como resonancia y/o apéndice sintomático del cuerpo histérico femenino en el Melodrama. Cfr. Nowell-Smith. Op. Cit., p.116. 


\section{Referencias bibliográficas}

ButLer, Alison. Women's Cinema (The contested screen). Short cuts, Wallflower, London/New York, 2002.

ButLER, Judith. Gender Trouble (Feminism and the Subversión of Identity). Routledge, London/New York, 1999.

Cook, Pam. «Melodrama and the Women's Picture», en Aspinall, Sue \& MurPHY, Robert (eds). Gainsborough Melodrama. BFI Dossier, № 18, British Film Institute, 1983, pp. 14-27.

De Lauretis, Teresa. Alicia ya no (Feminismo, Semiótica, Cine). Cátedra, Madrid, 1992.

De Miguel, Casilda; ItuARTe, Leire, AgIRre, Katixa. «Spanish Cinema through its Women Directors: 1995-2005», en Esteban, Mari Luz; Amurrio, Mila (eds). Feminist Challenges in the Social Sciences (Gender Studies in the Basque Country). Current Research Series, No. 2, Center for Basque Studies, University of Nevada, Reno/UPV/EHU, 2010, pp. 101-113.

DoAne, Mary Ann. The Desire to Desire (The Woman's Film of the 1940s). Indiana University Press, Bloomington\&Indianapolis, 1987.

— «The Woman's Film (Possession and Address)», en Gledhill, Christine (ed). Home is Where the Heart is (Studies in Melodrama an the Woman's Film). BFI, London, 1987, pp. 283-298.

ELSAESSER, Thomas. "Tales of Sound and Fury (Observations on the Family Melodrama)». Monogram, $\mathrm{n}^{\circ}$ 4, (1972), pp.2-15.

GLEDHILL, Christine (ed). Home is Where the Heart is (Studies in Melodrama an the Woman's Film). BFI, London, 1987.

KRISTEVA, Julia. Sol negro. Depresión y melancolía. Monte Avila Editores Latinoamericana, Venezuela, 1997.

KuHN, Annete. «Women's Genres (Melodrama, Soap Opera and Theory)», en GLEDHILl, Christine (ed). Home is Where the Heart is (Studies in Melodrama an the Woman's Film). BFI, London, 1987, pp. 339-349.

MerCER, John \& SHIngler, Martin. Melodrama, (Genre, Style, Sensibility). Short Cuts, Wallflower, London/New York, 2004.

MulveY, Laura. «Notes on Sirk and Melodrama» (1977/78), en GLEDHILL, Christine (ed). Home is Where the Heart is (Studies in Melodrama an the Woman's Film). BFI, London, 1987, pp. 75-79.

Nowell-Smith, Geoffrey. «Minelli and Melodrama». Screen, 18, 2, (1977), pp. 113-119.

Silverman, Kaja. The Acoustic Mirror (The Female Voice in Psychoanalysis and Cinema). Indiana University Press, Bloomington/Indianapolis, 1988. 